\title{
Armonización del derecho cooperativo en América Latina: desafíos y oportunidades
}

\author{
Dante Cracogna \\ Universidad de Buenos Aires
}

Sumario: I. Introducción. II. El Proyecto de Ley Marco para las Cooperativas de América Latina. III. Características del Proyecto de Ley Marco. IV. Proceso de actualización. V. Contenidos y alcances de la Ley Marco. VI. Conclusiones.

Resumen: Este trabajo reproduce la ponencia presentada por el autor en el Foro organizado por SGECOL (Grupo de Estudio sobre el Derecho Cooperativo Europeo) realizado en Manchester el 31 de octubre de 2012 en el marco de las actividades de cierre del Año Internacional de las Cooperativas organizadas por la Alianza Cooperativa Internacional.

El trabajo versa sobre el Proyecto de Ley Marco para las Cooperativas de América Latina elaborado por un grupo de expertos de diferentes países de la región por encargo de la Organización de las Cooperativas de América (OCA) en 1988, el cual ejerció apreciable influencia en la posterior legislación continental. Seguidamente aborda el proceso de actualización del documento llevado a cabo por la Alianza Cooperativa Internacional para las Américas (ACI Américas) veinte años más tarde. Finalmente, trata sobre los objetivos del documento, los principios que lo inspiran, sus principales institutos y sus perspectivas en el marco de la reforma de la legislación cooperativa de los países latinoamericanos.

Palabras clave: Legislación cooperativa, América Latina, ACl Américas, Ley Marco para Cooperativas.

Abstract: This article reproduces the presentation delivered by the author at the Open Seminar organized by SGECOL (Study Group on European Cooperative Law) held in Manchester on the 31st October 2012 within the activities organized by the International Cooperative Alliance on the closing of the International Year of the Cooperatives.

The article deals about the Project of the Framework Law for Cooperatives in Latin America prepared by a group of experts convoked by the Organization of the Cooperatives of America (OCA) in 1988 which had a remarkable influence in the reform of the cooperative laws of different countries of the region. Afterwards, the updating process of the document undertook by the Interna- 
tional Cooperative Alliance for the Americas (ICA Americas) twenty year later is addressed. Finally, the underlying principles, the main aspects of the document and its perspectives for the development of the Cooperative Law in Latin America are considered.

Key words: Cooperative legislation, Latin America, ICA Americas, Framework Law for Cooperatives. 


\section{Introducción}

Agradezco la oportunidad de presentar la experiencia latinoamericana sobre la armonización del Derecho Cooperativo en este Seminario sobre el Derecho Cooperativo en Europa. El diálogo de las regiones contribuye a la construcción de un mejor derecho y al progreso de la ciencia jurídica en el mundo globalizado. Cabe esperar que encuentros como éste, realizados en distintas partes del mundo, produzcan resultados favorables para el acercamiento de las instituciones jurídicas, cuyo primer paso es el mejor conocimiento de ellas y de sus problemas a través del diálogo entre los estudiosos de la materia.

El caso de América Latina presenta algunas características singulares puesto que se trata de una región compuesta por países de una tradición jurídica similar: el derecho continental europeo de raíz romanista. Esos países tienen asimismo una historia común pues todos provienen del mismo tronco hispánico, salvo Brasil cuyo origen es portugués, y han alcanzado su independencia prácticamente al mismo tiempo. El idioma de la región es el español, con la excepción de Brasil, lo cual constituye un rasgo de comunidad cultural muy relevante. En ese contexto, no es de extrañar que las instituciones jurídicas fundamentales exhiban caracteres similares.

La legislación cooperativa en la región comenzó a aparecer a fines del siglo XIX formando parte de los códigos de comercio en México y Argentina. En el período entre ambas guerras se intensificó el proceso legislativo, el cual se completó después de la Segunda Guerra en casi todos los países. En las décadas posteriores tuvo lugar la revisión y reforma de muchas de ellas.

Los Congresos Continentales de Derecho Cooperativo convocados por la Organización de las Cooperativas de América (OCA) que se llevaron a cabo en diferentes países a partir de 1969 sirvieron para el intercambio de conocimientos y experiencias entre los especialistas de los diferentes países y sus conclusiones brindaron importantes materiales que contribuyeron a delinear orientaciones comunes del Derecho Cooperativo en la región ${ }^{1}$.

1 Los documentos finales de estos congresos se encuentran en: I Congreso Continental de Derecho Cooperativo, Universidad de los Andes, Mérida, 1969; Carta Jurídica de San Juan, Administración de Fomento Cooperativo, San Juan, 1976; Anales del III Congreso Continental de Derecho Cooperativo, Idelcoop-Intercoop, Buenos Aries, 1987 y Derecho Cooperativo. Tendencias actuales en Latinoamérica y la Comunidad Económica Europea, Antropos, Bogotá, 1993. 


\section{II: El Proyecto de Ley Marco para las Cooperativas de América Latina}

Los congresos mencionados y las actividades realizadas por la comisión jurídica de la OCA llevaron a advertir la conveniencia de elaborar un documento que sirviera de orientación para actualizar y perfeccionar la legislación cooperativa de los países latinoamericanos. Consiguientemente, en 1987 OCA convocó a un grupo de expertos de distintos países del Continente y a algunos especialistas europeos a una reunión técnica realizada en Bolivia. En esa ocasión se efectuó un amplio relevamiento crítico de la legislación continental, luego del cual se determinaron los aspectos de mayor relevancia para ser tenidos en cuenta en ella. Resultado de ese trabajo fue el documento «Bases para la legislación cooperativa en los países de América Latina»².

El mencionado documento fue ampliamente difundido a fin de recibir opiniones de especialistas y dirigentes del movimiento cooperativo de los diferentes países. Finalizado el período de consulta, se realizó una nueva reunión de expertos en la que se consideró un borrador del Proyecto de Ley Marco para las Cooperativas de América Latina que recogía las opiniones recibidas. Dicho borrador fue asimismo circulado para consulta.

Posteriormente los coordinadores del grupo de expertos tuvieron a su cargo dar redacción final al Proyecto y preparar la correspondiente justificación de cada una de sus disposiciones. De esa forma se llegó a la culminación del proceso que tuvo lugar en oportunidad de celebrarse la Asamblea Continental de la OCA en Bogotá en noviembre de 1988 en la cual obtuvo aprobación definitiva ${ }^{3}$.

A partir de ese momento se realizó una intensa labor de difusión del Proyecto de Ley Marco en todo el Continente mediante publicaciones, reuniones, seminarios y presentaciones en los parlamentos nacionales, la cual dio como resultado que el documento alcanzara una significativa in-

2 Este documento, redactado por Carlos Torres y Torres Lara y Dante Cracogna, quienes actuaron como coordinadores del grupo de expertos, fue publicado en la $R e$ vista de la Cooperación Internacional, órgano de la Alianza Cooperativa Internacional, No 1-1988, Intercoop, Buenos Aires.

3 El texto final con la justificación respectiva fue publicado como «Documento Especial N 3», América Cooperativa, OCA, Bogotá, 1988. Sobre los antecedentes y contenido pueden consultarse: Cracogna, Dante, «Un intento de armonización de la legislación cooperativa: el Proyecto de Ley Marco para las Cooperativas de América Latina», Anuario de Estudios Cooperativos, Instituto de Estudios Cooperativos, Universidad de Deusto, 1989, p. 129 y ss; Montolío, José María, Legislación cooperativa en América Latina. Situación, Derecho comparado y Proceso de armonización, Ministerio de Trabajo y Seguridad Social, Madrid, 1990, p. 267 y ss. 
fluencia en la renovación y actualización de la legislación cooperativa regional. Ya la ley colombiana $N^{\circ} 79$, que se sancionó en forma prácticamente contemporánea con el Proyecto de Ley Marco, acusa claramente la influencia de éste. Y desde entonces, extendió su influencia sobre las nuevas leyes de cooperativas que se fueron sucediendo: Honduras; Paraguay; Puerto Rico; México; Uruguay; Panamá; Nicaragua y Venezuela. Por otra parte, el Proyecto de Ley Marco estimuló el desarrollo de los estudios teóricos y el progreso del Derecho Cooperativo en general.

\section{Características del Proyecto de Ley Marco}

Si bien el Proyecto de Ley Marco tiene la estructura de un verdadero texto legislativo, no se elaboró con el propósito de convertirse en un texto uniforme o modelo para ser reproducido en los países de la región. Como su nombre lo indica, trata de establecer un marco que sirva de orientación general en la materia sobre la base de contenidos fundamentales de reconocida eficacia y con amplio margen para su adecuación a las diferentes circunstancias de cada país.

El documento no tiene la prensión de establecer rígidas uniformidades pues reconoce las diversidades nacionales, propias de sus características históricas y culturales. De allí que solamente trate de brindar pautas de orientación sobre aquellos aspectos en los que existen criterios comunes fundados en la peculiar naturaleza de las cooperativas y en las experiencias más relevantes de desarrollo cooperativo continental. De todas maneras, juega a favor del Proyecto de Ley Marco la señalada circunstancia de que la tradición jurídica latinoamericana en general tiene rasgos comunes.

Por otro lado, se reconoce que el Proyecto de Ley Marco no se debe considerar como un logro definitivo sino como una aproximación a un tema que por su naturaleza es complejo y susceptible de modificaciones. Por lo tanto, se trata de un documento perfectible y actualizable para dar respuesta adecuada a las cambiantes necesidades del desarrollo cooperativo regional.

\section{Proceso de actualización}

Transcurridos veinte años desde la aprobación del Proyecto de Ley Marco, y habiendo entretanto cesado la actividad de la OCA, la Alianza Cooperativa Internacional para las Américas ( $\mathrm{ACl}$ Américas) decidió encarar su actualización. Para ello tuvo en consideración los importantes 
cambios que se habían producido en la región y en el mundo que hacían necesario realizar una puesta al día a fin de que el documento siguiera siendo útil para la finalidad originariamente prevista. Además de las inéditas circunstancias del mundo globalizado se habían emitido relevantes pronunciamientos de organismos internacionales que exigían ser tomados en consideración dentro del marco legislativo ${ }^{4}$.

En primer lugar, el Congreso del Centenario de la $\mathrm{ACl}$, realizado en Manchester en 1995, había aprobado la Declaración sobre la Identidad Cooperativa que contenía una reformulación de los principios cooperativos que debía ser tenida en cuenta en cualquier proyecto de legislación puesto que su contenido informa toda manifestación cooperativa.

A ello se suma la Resolución 56/114 sobre las cooperativas en el desarrollo social aprobada por la Asamblea General de la Organización de las Naciones Unidas en diciembre de 2001 cuyo Anexo —además de tratar otros aspectos - hace específica referencia a cuestiones de orden legislativo relacionadas con las cooperativas ${ }^{5}$.

Otro documento de significativa importancia emanado de un organismo internacional es la Recomendación 193 sobre promoción de las cooperativas aprobada por la Organización Internacional del Trabajo (OIT) en 2002 en sustitución de la anterior Recomendación 127 de 1966. Esta Recomendación constituye el documento más completo y abarcativo en la materia y contiene numerosas disposiciones relacionadas con la legislación y las políticas públicas en materia de cooperativas.

Estos documentos, como señala Hagen Henrÿ, constituyen el núcleo del Derecho Público Internacional Cooperativo que no puede ser ignorado por las legislaciones nacionales ${ }^{7}$.

A su vez, la Asamblea General de ACI realizada en Seúl en 2001 recomendó unos Lineamientos de Legislación Cooperativa elaborados

4 Cracogna, Dante, «Nueva versión de la Ley Marco para las cooperativas de América Latina», Revista Jurídica de Economía Social y Cooperativa, N²0, CIRIEC-España, Valencia, 2009, p. 183 y ss.

5 El Anexo de esta Resolución, titulado "Guidelines aimed at creating a supportive environment for the development of cooperatives», constituye un valioso vademécum de orientación acerca de las políticas públicas en materia de cooperativas que comprende diversos aspectos vinculados con la legislación cooperativa.

6 Cabe señalar que la Recomendación 193 tiene un alcance mucho más amplio que la anterior Recomendación 127 pues ésta se hallaba referida solamente a los países en desarrollo en tanto que la primera se refiere a todos. Por otra parte, es notable que la actual Recomendación fue aprobada en forma prácticamente unánime por los representantes de los gobiernos, de los trabajadores y de los empresarios que forman la Asamblea General de la OIT.

7 Henrÿ, Hagen, Guidelines for Cooperative Legislation, 2nd revised edition, ILO, Geneva, 2005, p. 5. 
sobre la base de estudios promovidos por la OIT, los cuales contienen importantes orientaciones para el mejoramiento de la legislación específica ${ }^{8}$ Una vez que la ACI Américas resolvió encarar la tarea de actualización obtuvo el apoyo del Servicio de Cooperativas de la OIT que brindó su colaboración técnica y su amplia experiencia en materia de legislación cooperativa.

A efectos de realizar la tarea se designó una comisión de expertos representativos de las diferentes regiones del continente latinoamericano: Cono Sur; Area Andina; Centroamérica y el Caribe, quienes contaron con la colaboración del Jefe del Servicio de Cooperativas de la OIT.

Esta tarea se llevó a cabo a lo largo de 2007 arribándose a un texto provisorio que fue sometido a la XV Conferencia Regional de la $\mathrm{ACl}$ Américas en octubre de ese mismo año. Los miembros de la comisión de expertos recogieron sus aportes para la posterior elaboración de una nueva versión que fue ampliamente circulada entre las organizaciones asociadas a la $\mathrm{ACl}$ Américas, universidades e institutos especializados. Con todos esos insumos se llegó a un borrador final que fue finalmente aprobado en la reunión de la $\mathrm{ACl}$ Américas realizada en julio de 2008. A continuación fue oficialmente publicado ${ }^{9}$ y quedó en condiciones de ser difundido para que cumpliera su cometido.

\section{Contenido y alcances de la Ley Marco}

Como se dijo, el documento no es una Ley Modelo entendida en el sentido de una elaboración para ser adoptada en diferentes países mediante el sencillo expediente de su copia o transcripción ${ }^{10}$. Por el contrario, se trata de un documento pensado para servir de orientación en

8 Estos «Lineamientos para la legislación cooperativa» fueron elaborados por Hagen Henrÿ y están publicados en la Revista de la Cooperación Internacional, № 2-2001, Buenos Aires, p. 67 y ss.

9 Alianza Cooperativa Internacional para las Américas, Ley Marco para las cooperativas de América Latina, ACl Américas, San José (Costa Rica), 2009, 45 págs. Existen también versiones en inglés y portugués.

10 Un caso paradigmático de Ley Modelo es el que fue elaborado por la British Colonial Office en1946 sobre la base de la experiencia de sus colonias y, especialmente, de la India. Cfr. Surridge, J.B. and Digby, Margaret, A Manual of Co-operative Law and Practice, $3^{\text {rd }}$ edition, W. Heffer and Sons, Cambridge, 1967, p. 121 y ss. También se refiere a ella Campbell, W.K.H, Practical Co-operation in Asia and Africa, W. Heffer and Sons, Cambridge, 1959, P. 19 y ss. En la actualidad, la Ley Uniforme relativa al derecho de las sociedades cooperativas, aprobada por la OHADA (Organización para la Harmonización en Africa del Derecho de los Negocios), publicada en el Diario Oficial de la OHADA, Yaoundé, Cameroun, 15.2.11. 
la renovación de la legislación cooperativa regional; es una contribución puesta a disposición de los movimientos cooperativos y de los legisladores nacionales como herramienta de trabajo para actualizar las leyes de la materia, algunas de las cuales se hallan considerablemente atrasadas en el tiempo.

La Ley Marco está elaborada como una ley general para toda clase de cooperativas, afirmando de esa manera la unidad fundamental del movimiento cooperativo con independencia de sus diversos sectores o ramas específicas. Contiene solamente un capítulo con algunas disposiciones especiales para ciertas clases de cooperativas que por su particular naturaleza las requieren: cooperativas de trabajo asociado; bancos cooperativos, cooperativas de ahorro y crédito y de seguros; cooperativas de vivienda; cooperativas escolares y juveniles. Esta ley general podría, en caso de resultar necesario, complementarse con leyes especiales para determinadas clases de cooperativas - como suele suceder en algunos países con las cooperativas de ahorro y crédito- pero que regulen solamente los aspectos operativos propios de ellas dejando las demás cuestiones sujetas a la ley general.

No se tratan medidas de fomento o de promoción por cuanto se considera que ellas quedan libradas a la decisión de cada país dentro del marco de su respectiva política económica y social general.

Se tuvo especial cuidado de que la Ley Marco cubriera adecuadamente todos los aspectos fundamentales relacionados con la regulación de las cooperativas de manera de evitar, en lo posible, la necesidad de dictar reglamentaciones. Cuando resulta necesario el dictado de normas reglamentarias el texto lo dice expresamente, entendiéndose que en los demás casos las disposiciones legales resultan directamente aplicables procurando de esa manera evitar las reglamentaciones que los funcionarios gubernamentales suelen dictar yendo, incluso, más allá de la propia ley ${ }^{11}$.

En cambio, se deja amplio espacio librado a las disposiciones estatutarias, de manera que cada cooperativa pueda establecerlas conforme con sus propias necesidades y con considerable margen de autonomía.

Se organizan las disposiciones en 102 artículos agrupados en doce capítulos utilizando una terminología generalmente aceptada en los países de la región y consistente con la doctrina cooperativa a fin de que cada instituto refleje apropiadamente su naturaleza.

11 Caso representativo puede considerarse el de Ecuador, cuya anterior Ley de Cooperativas tenía 160 artículos, en tanto que su decreto reglamentario original contenía más de 230 , a lo que se sumaban los numerosos reglamentos posteriores, dictados mediante decretos o resoluciones ministeriales. 
El texto comienza con las disposiciones generales que caracterizan a las cooperativas para luego ocuparse, a lo largo de los sucesivos capítulos, de la constitución; los socios; el régimen económico; los órganos sociales (asamblea; consejo de administración y junta de vigilancia); la integración entre cooperativas; la disolución y la liquidación; normas para ciertas clases de cooperativas en particular; la autoridad de contralor o fiscalización y, finalmente, el organismo encargado de la política cooperativa.

En cuanto a su presentación, tiene las características formales de un texto legal donde cada artículo va precedido de un subtítulo que anuncia su contenido y seguido de una breve justificación que explica el sentido y alcance de la norma de manera de hacer más fácil su comprensión.

Por último, cabe señalar que los principios básicos inspiradores de la Ley Marco son: a) consolidación de la identidad cooperativa; b) fortalecimiento del sentido empresarial y c) afirmación de la autonomía de las cooperativas frente al Estado.

\section{Conclusiones}

La Ley Marco significa un aporte de singular importancia para la armonización de la legislación cooperativa en el Continente. Tiene el mérito de haber sido elaborada y actualizada con la participación de expertos de diferentes países tomando en cuenta la experiencia cooperativa regional, los desarrollos más relevantes del derecho comparado y los documentos de los organismos internacionales. Finalmente, fue sometida a una amplia consulta antes de su aprobación y difusión. Su influencia se advierte claramente en la legislación y en la teoría del Derecho Cooperativo Latinoamericano.

A fin de lograr una mayor eficacia en el cumplimiento del objetivo de la Ley Marco, la ACl Américas promovió su adopción por el Parlamento Latinoamericano, organismo que si bien no cuenta con atribuciones legislativas puede formular recomendaciones a los parlamentos nacionales de los países de la región. Dicho organismo, luego del estudio y dictamen favorable de la comisión respectiva, le dio aprobación en su sesión plenaria de fines de 2012, lo cual permite considerar que se intensificará su influencia en la legislación de los países latinoamericanos. 